\title{
Assessment of copy number variations in the brain genome of schizophrenia patients
}

\author{
Miwako Sakai ${ }^{1,2}$, Yuichiro Watanabe ${ }^{2}$, Toshiyuki Someya ${ }^{2}$, Kazuaki Araki ${ }^{1}$, Masako Shibuya ${ }^{2}$, Kazuhiro Niizato ${ }^{3}$, \\ Kenichi Oshima ${ }^{3}$, Yasuto Kunii ${ }^{4}$, Hirooki Yabe ${ }^{4}$, Junya Matsumoto ${ }^{4}$, Akira Wada ${ }^{4}$, Mizuki Hino ${ }^{4}$, Takeshi Hashimoto ${ }^{5}$, \\ Akitoyo Hishimoto ${ }^{5}$, Noboru Kitamura ${ }^{5}$, Shuji Iritani ${ }^{3,6}$, Osamu Shirakawa ${ }^{5,7}$, Kiyoshi Maeda ${ }^{5,8}$, Akinori Miyashita ${ }^{9}$, \\ Shin-ichi Niwa ${ }^{4}$, Hitoshi Takahashi ${ }^{10}$, Akiyoshi Kakita ${ }^{10}$, Ryozo Kuwano ${ }^{9}$ and Hiroyuki Nawa ${ }^{1 *}$
}

\begin{abstract}
Background: Cytogenomic mutations and chromosomal abnormality are implicated in the neuropathology of several brain diseases. Cell heterogeneity of brain tissues makes their detection and validation difficult, however. In the present study, we analyzed gene dosage alterations in brain DNA of schizophrenia patients and compared those with the copy number variations (CNVs) identified in schizophrenia patients as well as with those in Asian lymphocyte DNA and attempted to obtain hints at the pathological contribution of cytogenomic instability to schizophrenia.

Results: Brain DNA was extracted from postmortem striatum of schizophrenia patients and control subjects $(n=48$ each) and subjected to the direct two color microarray analysis that limits technical data variations. Disease-associated biases of relative DNA doses were statistically analyzed with Bonferroni's compensation on the premise of brain cell mosaicism. We found that the relative gene dosage of 85 regions significantly varied among a million of probe sites. In the candidate CNV regions, 26 regions had no overlaps with the common CNVs found in Asian populations and included the genes (i.e., ANTXRL, CHST9, DNM3, NDST3, SDK1, STRC, SKY) that are associated with schizophrenia and/or other psychiatric diseases. The majority of these candidate CNVs exhibited high statistical probabilities but their signal differences in gene dosage were less than 1.5-fold. For test evaluation, we rather selected the 10 candidate CNV regions that exhibited higher aberration scores or larger global effects and were thus confirmable by PCR. Quantitative PCR verified the loss of gene dosage at two loci (1p36.21 and 1p13.3) and confirmed the global variation of the copy number distributions at two loci (11p15.4 and 13q21.1), both indicating the utility of the present strategy. These test loci, however, exhibited the same somatic CNV patterns in the other brain region.
\end{abstract}

Conclusions: The present study lists the candidate regions potentially representing cytogenomic CNVs in the brain of schizophrenia patients, although the significant but modest alterations in their brain genome doses largely remain to be characterized further.

Keywords: CNV, Caudate, Genome instability, Schizophrenia, Somatic mutation

\section{Background}

Copy number variation (CNV) is defined as a deletion or duplication/multiplication of a genomic fragment spanning more than $1 \mathrm{~kb}$ when compared to a reference genome [1-3]. Approximately 37,000 sites of common CNVs have been identified in the human genome and they occupy $12 \%$ of the entire genome $[4,5]$. The genome-wide

\footnotetext{
*Correspondence: hnawa@bri.niigata-u.ac.jp

'Department of Molecular Neurobiology, Brain Research Institute, Niigata University, 1-757, Asahimachi-dori, 951-8585 Niigata, Japan

Full list of author information is available at the end of the article
}

association studies (GWAS) on schizophrenia analyzed DNA which was isolated from peripheral lymphocytes and have identified risk $\mathrm{CNV}$ sites, some of which are not present in the patients' parents [6-9].

Somatic mosaicism of genome sequences and structures have recently drawn particular attention [10-12]. Nearly $30 \%$ of developing brain cells in human are reported to harbor aberrant chromosomal compositions [13, 14]. In addition, there are significant genomic differences in somatic cells between monozygotic twins and among tissues [15-18]. Accordingly, aberrant cytogenomic variations in 
human brain are implicated in neurodegenerative and neurodevelopmental disorders such as Alzheimer's disease, amyotrophic lateral sclerosis, and Huntington's diseases [19-25]. It is an open question whether the brain-specific somatic mutation or $\mathrm{CNV}$ might also contribute to the etiology or neuropathology of schizophrenia [26-28].

To obtain hints at the above question, we prepared DNA from the brain tissue of 48 schizophrenia patients and 48 control subjects. Labeling brain DNA samples, we directly applied those to Agilent $1 \mathrm{M}$ comparative genomic hybridization $(\mathrm{CGH})$ arrays to measure relative gene doses without the use of reference genome. This direct comparison through the case-control pairing reduces technical data deviations and enhances the statistical power of detection [29, 30]. With the potential genomic mosaicism of heterogeneous brain cell mixtures, we expected that the target genome could be diluted with normal DNA from the off-target cells and thus assumed non-integer values of CNVs in this analysis [31]. Technical limitations of this approach are further discussed below.

\section{Results}

The striatum contain neural stem cells that proliferate throughout human life and carries somatic mutation in its mitochondrial genome [32, 33]. Therefore, we hypothesized that the striatum may be a potential candidate region that would exhibit somatic mosaicism in brain genome structures. DNA was extracted from postmortem striatum of patients with chronic schizophrenia $(n=48)$ and agematched controls who had no history of neuropsychiatric disorders $(n=48)$ (Additional file 1: Table S1). Although there were significant differences in postmortem intervals (PMIs) between groups, there was no detectable difference in DNA quality (data not shown). All other indices were indistinguishable between schizophrenia patients and control subjects. A DNA sample was randomly picked from each group, paired to a sample in the other group, and subjected to two-color competitive CGH analysis with 1 M SurePrint G3 Human CGH Microarrays.

We applied the ADM-2 algorithm to the CGH signals of individual microarray probes (nearly 1 million) and searched for the primary candidate $\mathrm{CNV}$ loci associated with schizophrenia. A flowchart of the present study design is shown in Additional file 1: Figure S1. We chose1381 chromosomal loci that exhibited large group differences in gain/loss calls (Selection 1). In each probe site located on the primary candidate loci, we plotted the distribution of $\log 2$ signal ratios from 48 sets of microarray analyses and tested the null hypothesis that the mean $\log 2$ signal ratios was zero, indicating that the two groups were indistinguishable (Selection 2). We calculated total probabilities and averaged log2 signal ratios for individual candidate loci and judged their statistical significance with Bonferroni's correction. The number of the candidate loci maintaining the statistical significance through Selection 2 was reduced to 85 (Details in Additional file 1: Table S2).

Positive CNV loci were found in almost all chromosomes except chromosome 17 and 21 (Fig. 1). Individual loci covered 1-746 probe sites $(3-2200 \mathrm{~kb})$ and exhibited average $\log 2$ ratios of -1.46 to +0.63 (i.e., odds ratio $(\mathrm{OR})=$ $0.36-1.55)$. A majority of the average $\log 2$ ratios were between -0.59 to +0.59 (i.e., $<1.5$-fold differences) and only 4 loci showed more than 1.5-fold differences in array $\mathrm{CGH}$ signals. A genomic region spanning from $6 \mathrm{p} 22.2$ to 6 p21.32 contained six CNV loci and included genes for the major histocompatibility complex that is highly associated with schizophrenia in GWAS [34]. Among the $85 \mathrm{CNV}$ loci in Selection 2, 59 loci were reported and 26 loci were not reported in the CNV study on leukocyte DNA samples of Asian populations (Additional file 1: Table S2) [2].

To validate the authenticity of the present procedure, we attempted to verify the above genome dosage changes of several candidate loci using quantitative polymerase chain reaction (qPCR). According to the following two criteria, we selected the test loci whose signal differences were larger between groups and could be detectable with the given accuracy of qPCR; (i) those exhibiting the large and consistent gain/loss calls across the limited sample pairs (from Selection 1) and (ii) the loci represented larger global effects shared in most of the schizophrenia samples (from Selection 2).

In the former category, the gene dosage of $\mathrm{Hs} 03385437$ (1p13.3), CC70L1J (1p36.21), Hs03318079 (Chr18:q22.1), Hs04794356 (4q24), Hs05080419 (9q22.2), and Hs07134106 (19p12) produced exclusive gain/loss calls in not less than four sample pairs. No discrepant calls were detected in any sample pairs. Using the same DNA pairs showing the difference in the penetrance call (Selection 1), we determined and confirmed the gene dosage of those DNA samples using qPCR. ANOVA detected significant gene dose differences at two loci (Hs03385437 and CC70L1J) between patient and control groups (Fig. 2).

In this measurement, we used RNaseP gene as an internal DNA dose control. Measured genome doses of the above regions appeared not to be integer levels in several control samples, potentially reflecting the cell mosaicism of the original tissues. We also extracted DNA from the prefrontal cortex of the same subjects of both groups and compared the genome doses of the above loci ( $\mathrm{Hs} 03385437$ and CC70L1J). We calculated the copy number ratio of the patient' DNA dosage to that of the control subject' dosage and compared these ratios between the brain regions. At both loci, almost all the copy number ratios were markedly lower than 1.0 except the C26:S34 pair, supporting our primary observation that the absolute gene dosages of these loci were decreased in the schizophrenia samples. However, copy number ratios did not significantly differ 


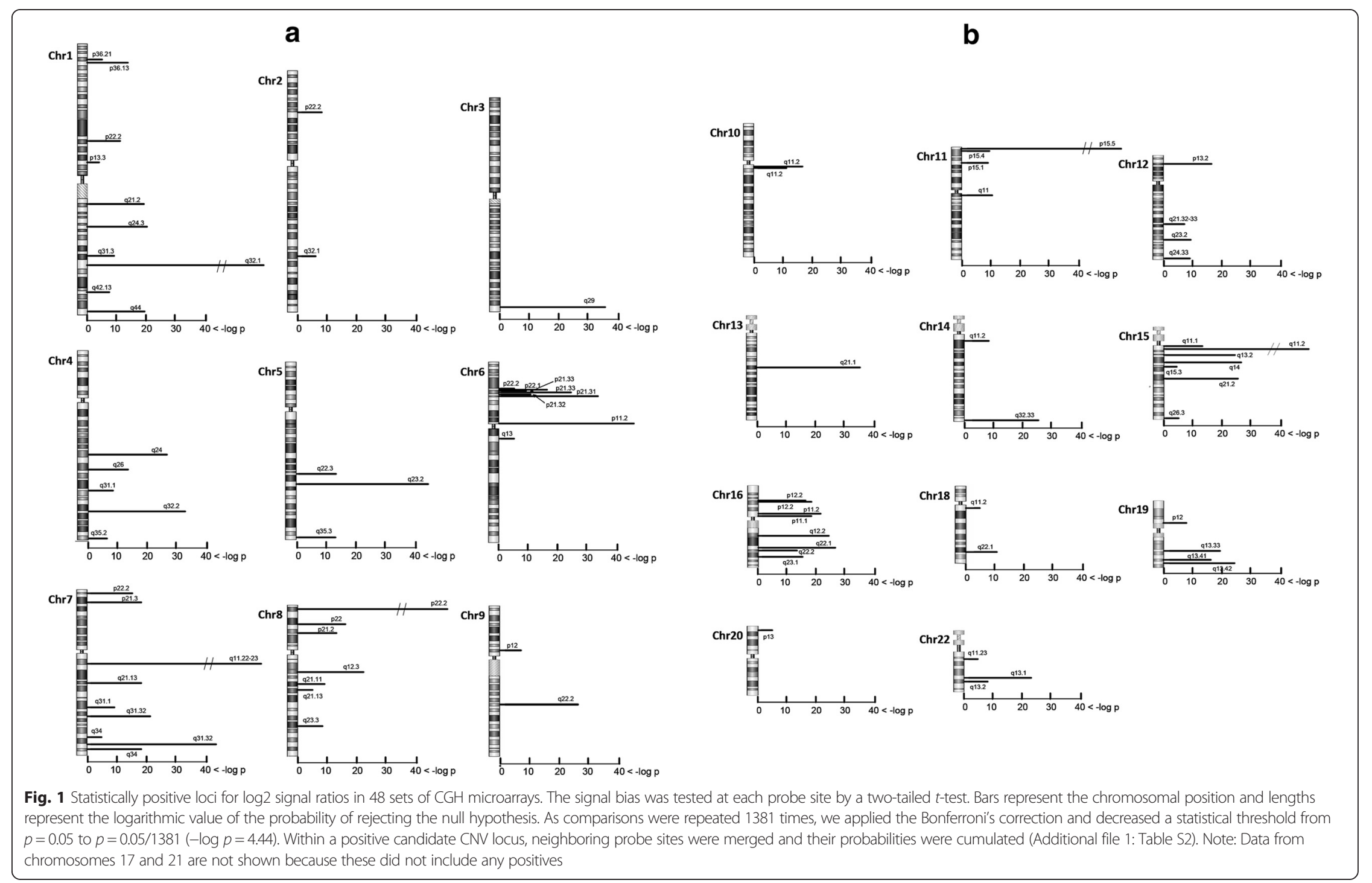




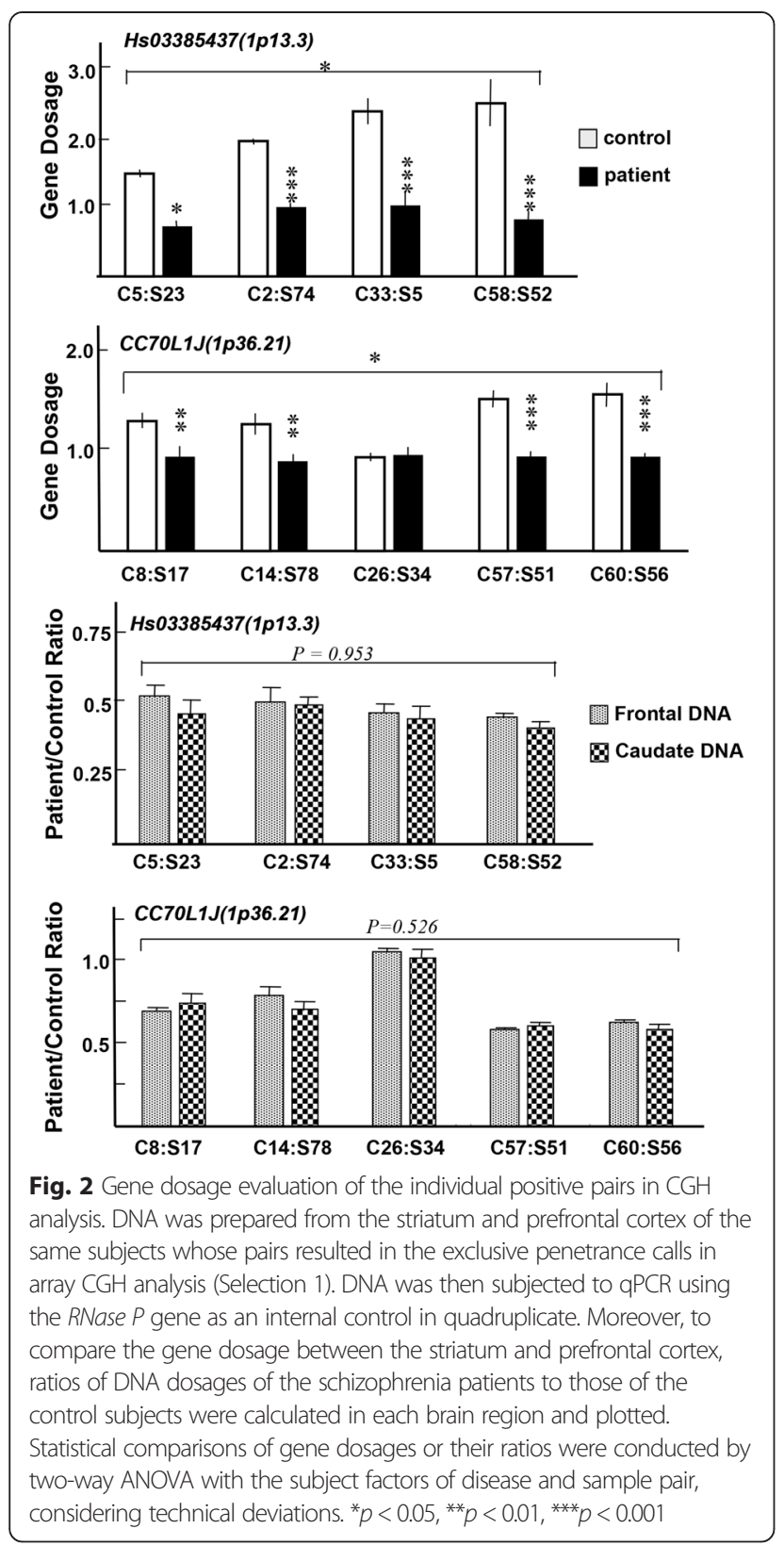

between these brain regions in any of the sample pairs (Fig. 2). At least at these two candidate loci, we failed to find evidence for a gene dosage difference between these brain regions.

In the latter category, Hs0358779 (6p22.1), Hs03265736 (7p21.3), Hs03765933 (11p15.4), and Hs03298358 (q21.1) exhibited higher log2 signal ratios and were thus subjected to the test evaluation. Gene dosage of these four loci were determined by qPCR using all the DNA samples in control and schizophrenia groups ( $\mathrm{n}=48$ each). Differences in gene dosages were replicated by qPCR for $\mathrm{Hs} 03765933$ and Hs03298358 (Fig. 3). In contrast to the data distributions of Fig. 2, almost all the values of the gene doses were located at the levels of integers but with several exceptions. These candidate $\mathrm{CNVs}$ appear to reflect the gene dosage differences of germinal origin.

\section{Discussion}

Several recent reports have indicated the neuropathological contribution of somatic CNV or DNA instability of the brain genome [19-28, 35-40]. In accordance with these findings, a small proportion few percent of brain cells are known to exhibit aneuploidy and carry large CNVs [13, 14, 41]. Aneuploidy is detected by fluorescence in situ hybridization (FISH) and appears to be increased by the onset of Alzheimer's disease [20,22]. The aneuploidy of chromosome 1, 18 and $\mathrm{X}$ was also identified in the brain of schizophrenia patients [21, 41]. Despite its advantages, FISH cannot be employed in exploratory investigations, unless the specific genome region of the CNV of interest is identified. Since bonafide genome structures from off-target cells could dilute the abnormal genome DNA population, more sensitive technologies remain to be developed, which detect low quantities of $\mathrm{CNV}$ in heterogeneous cell mixtures of the brain tissue [42, 43]. In the present study, we attempted to evaluate the efficacy of the CGH microarray technique to extract somatic CNVs in the postmortem brains of schizophrenia patients [42, 43].

With given semi-quantitative nature of the microarray technique, we applied statistics to the $1 \mathrm{M}$ array CGH results from 48 sample pairs. Using the high density CGH array and statistical approach, we found 85 candidate CNV loci in the present study; $59 \mathrm{CNV}$ loci are overlapped with the common $\mathrm{CNV}$ regions and the remaining 26 loci are not reported in peripheral leukocyte-derived DNA of Asian people $[2,44]$. Of note, the 26 candidate regions encode the seven genes that are associated with or implicated in schizophrenia or other psychiatric diseases; ANTXRL, CHST9, DNM3, NDST3, SDK1, STRC, and SKY (Additional file 1: Table S2). DNM3 in the candidate region of 1q24.3 is disruptively mutated in some of schizophrenia patients [45]. ANTXRL and CHST9 are located in the CNV regions associated with bipolar disorder and autism [46, 47]. NDST3 and STRC are the risk genes for schizophrenia and hearing impairment that are identified by GWAS, respectively [48, 49]. SDK1 and SKY are the genes whose expression levels are markedly altered in the brain of schizophrenia patients $[50,51]$. Accordingly, the present listing of the candidate brain CNVs is informative for future cytogenomic studies on schizophrenia [21, 41].

It was difficult for us to validate most of the abovementioned 85 candidate loci with qPCR analysis with the given small signal differences between groups (i.e., less than 1.5 -fold). Therefore, we selected the best 10 test loci that exhibited relatively large and/or wide effects on gene dosage. The six loci were chosen from Selection 1 as putative rare CNVs, which exhibited exclusive gain/loss calls in the 

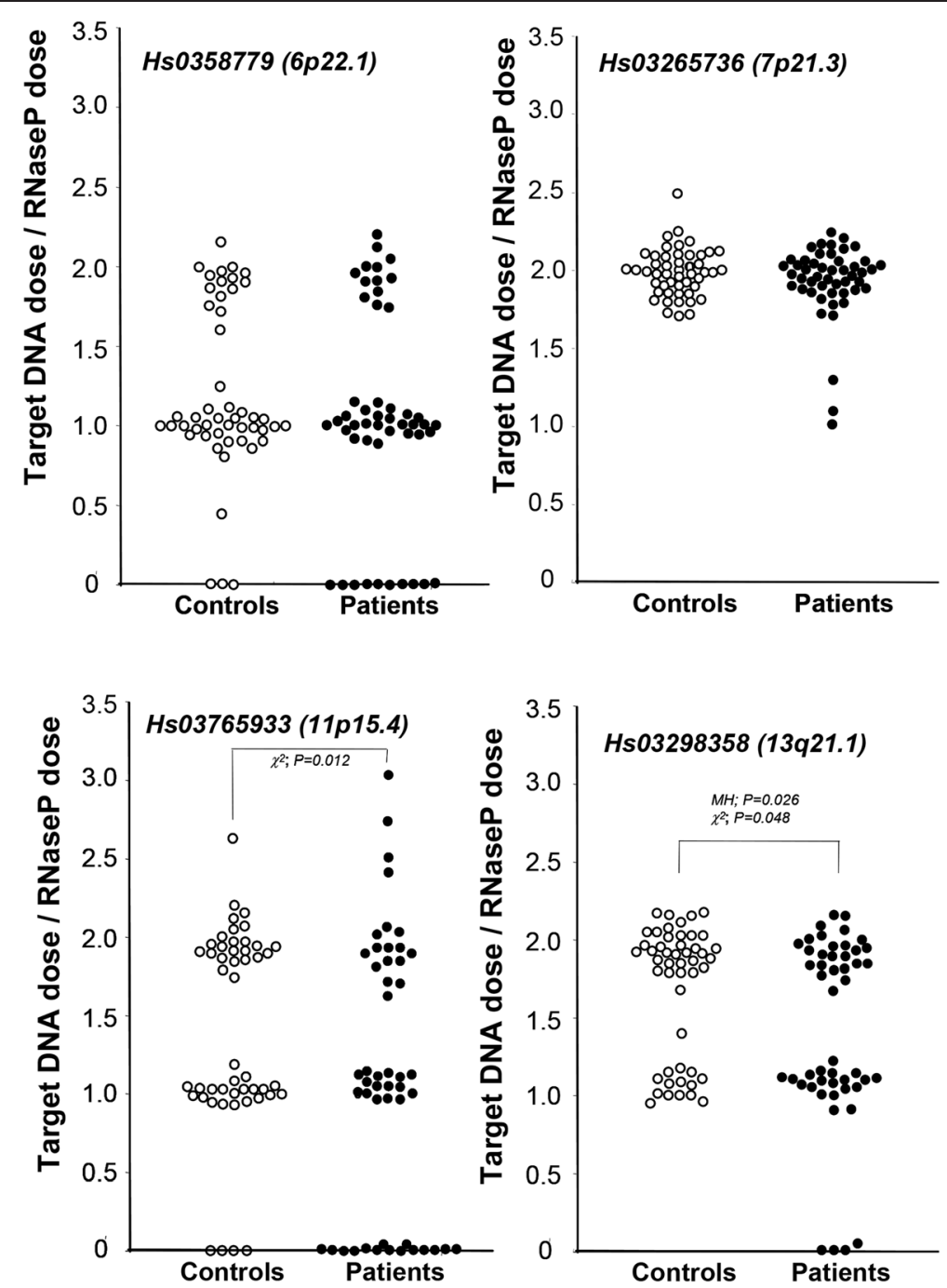

Fig. 3 qPCR evaluations of candidate CNV loci from Selection 2. Gene dosages of the candidate loci 6p22.1, 7p21.3, 11p15.4, 13q21.1, and the RNase P (internal control) were determined by qPCR using all sample DNAs and TaqMan probes (Additional file 1: Table S3). Individual gene dosages of 48 patients' or 48 controls' DNAs were plotted and compared between groups using the Chi-square and Mann-Whitney $U$ tests

limited number of samples. From Selection 2, the four loci were chosen as provisional common variants, which showed large effects and higher probability levels in the above parametric analysis. The qPCR analysis confirmed the schizophrenia-associated gene dosage differences at nearly half of the candidate CNV loci, suggesting the validity of the present strategy.

Unfortunately we had neither stored peripheral tissues nor information about these CNVs in peripheral DNA of the same subjects. To estimate the contribution of somatic CNVs to the present CNV listing, therefore, we were compelled to compare the gene dosages between the two brain regions or to search for their absence in the databases of Asian CNVs of leukocyte origin [2.44]. In the test PCR, however, we could not detect significant differences in gene dosages between the striatum and prefrontal cortex, at least, at these test $\mathrm{CNV}$ loci. If somatic CNVs were produced prior to neuroectodermal differentiation, there should be no difference between these two neural tissues, suggesting that the present comparison between these brain regions was inappropriate. Therefore, a comparative analysis of DNA from germinal cells of the same subjects will warrant this definitive conclusion [45].

Among the CNV candidate regions in Fig. 1, 26 candidate regions are not reported as the common CNVs of Asian populations [2, 44]. The majority of these loci exhibited high statistical significance with the probabilities of less than $10^{-100}$, such as $4 \mathrm{q} 35.2,6 \mathrm{p} 11.2,7 \mathrm{q} 11-12$, 11p15.4-15.5, and 15q11.2. In contrast, their CGH signal differences between patients and controls were markedly smaller ( $\mathrm{OR}=0.988-1.055)$. As discussed above, these candidate $\mathrm{CNV}$ loci include the peculiar genes that are 
implicated in schizophrenia [45-51]. These regions, which exhibited small signal differences, might represent more promising candidates of somatic $\mathrm{CNV}$ sites because the genome aberration of target cells is presumably diluted in the brain and should result in smaller ORs. However, such small differences in gene dosage should make the conventional qPCR verification more challenging with the given technical deviations [52]. To avoid target DNA dilution with cell mosaicism, single cell qPCR or FISH may be more beneficial in theory [20-22, 43]. However, it would be difficult to independently perform microdissection of hundreds of cells and perform single-cell analysis unless the target cell population is identified with molecular markers and its sensitivity of gene detection is high enough. FISH also requires properly fixed and processed brain tissues of the same subjects. With the given technical difficulties, therefore, we have been unable to verify these small variations.

\section{Conclusion}

The present $\mathrm{CGH}$ analysis lists the potential candidate regions of somatic CNVs associated with schizophrenia, although most of those exhibited the modest but highly significant alterations in brain genome doses. Future studies aim to develop more elaborate techniques for somatic genome mosaicism and to verify the schizophreniaassociated cytogenomic instability in the above CNV candidates [53-56].

\section{Methods}

\section{Ethical approval}

The study was approved by Niigata University Medical Ethics Committee (No. 683). The use of postmortem brain tissues was authorized by the Matsuzawa Hospital Ethics Committee, Kobe University Medical Ethics Committee, Fukushima Medical University Ethics Committee, and Niigata University Medical Ethics Committee. The families of the control and schizophrenia patients provided written informed consent to allow the use of brain tissues for pathological investigations.

\section{Brain tissue}

Postmortem brain tissue was collected from patients with chronic schizophrenia (30 men, 18 women; mean age, $64.5 \pm 12.5$ years old) and from age-matched control subjects (30 men, 18 women; mean age, $64.2 \pm 12.0$ years old), with no history of neuropsychiatric disorders (Additional file 1: Table S1). The diagnosis of schizophrenia was confirmed by examining the patient's report according to DSM-III or DSM-IV categories (American Psychiatric Association). Postmortem brains of schizophrenia patients were collected at Matsuzawa Hospital, Kobe University, Fukushima Medical University and Niigata University, while those of control subjects were collected at Niigata
University. In brief, the left cerebral hemisphere was fixed in formalin for diagnostic examination and the right hemisphere was frozen at $-80{ }^{\circ} \mathrm{C}$. Tissue samples were taken from postmortem brains that did not exhibit neurodegenerative abnormalities by conventional pathological staining (data not shown). The striatum (caudate) was identified in frozen coronal slices according to a human brain atlas. All tissues were collected and stored according to the principles of the Declaration of Helsinki, and tissue use was in compliance with the Human Tissue Act 2004.

\section{DNA extraction}

High molecular weight DNA was extracted by the guanidinium - phenol procedure (Gentra Pure Gene Tissue Kit, Qiagen, Tokyo, Japan) according to the manufacturer's protocol. Extracted DNA was quantified by spectrophotometry using a Nanodrop ND-2000 (Thermo Scientific Wilmington, DE, USA). Samples with absorbance ratios of A260/280 1.80 and A260/230 > 1.90, respectively, were regarded as sufficiently pure and suitable for $\mathrm{CGH}$ analysis. Some DNA samples were subjected to $1.0 \%$ agarose gel electrophoresis for quality control. Evidence of DNA degradation was not detected in randomly-picked DNA samples from patient or control groups (data not shown).

\section{Comparative genomic hybridization (CGH)}

Array-based CGH was performed by the manufacturer Takara Bio Dragon Genomics Center (Seta, Shiga, Japan). In brief, DNA (2 micro g) was fluorescentlabeled by random priming DNA synthesis in the presence of Cy3-dUTP (control group) or Cy5-dUTP (patient group) (Genomic DNA Enzymatic Labeling Kit; Agilent Technologies, Hachioji, Tokyo, Japan). DNA labeling efficiency was estimated by spectrophotometry (Nanodrop ND-2000 $0^{\circ}$ measuring optical absorbance at $260 \mathrm{~nm}$ for DNA, at $550 \mathrm{~nm}$ for Cy5, and at $649 \mathrm{~nm}$ for Cy3. Cy5- and Cy3-labeled DNAs were randomly paired, mixed, and hybridized to SurePrint G3 Human CGH Microarrays (1 M) in the presence of human Cot-1 DNA (Oligo aCGH/ChIP-on-chip Hybridization Kit, Agilent Technologies). Following hybridization for $24 \mathrm{~h}$, microarray slides were washed according to the manufacturer's instructions and immediately scanned on a DNA Microarray Scanner (Agilent Technologies). With the given limitation of the sample number, we took an advantage of the above direct comparison between case and control samples [57]. This approach allowed us to determine relative ratios of their gene dosages but not their absolute gene dosages. However this procedure decreased data deviations, compared with the $\mathrm{CGH}$ analysis utilizing two microarrays and reference genome DNA [30]. 


\section{Quantitative polymerase chain reaction (qPCR)}

To validate the results from the microarray experiments, we performed qPCR using TaqMan probes (Applied Biosystems, Foster City, CA) as described previously [32]. Gene dosages of the following genomic regions of interest were measured for the sample pair sets that exhibited the exclusive positive penetrance call with the Aberration Detection Method 2 (ADM-2) algorithm; CC70L1J (1p13.3), Hs03385437 (1p36.21), Hs04794356 (4q24), Hs05080419 (9q22.2), Hs03318079 (18q21.1), and Hs07134106 (19p12). Using all the samples, we also determined the gene dosages of the candidate CNV loci that exhibited lower probability scores by the global $t$-test analysis; $\mathrm{Hs} 03587795$ (6p22.1), Hs03265736 (7p21.3), Hs03765933 (11p15.4), and Hs03298358 (13q21.1). DNA sequences of TaqMan probes and PCR primers are shown in the Additional file 1 . We obtained cycle threshold (CT) values for the region of interest for each sample with $\mathrm{FAM}^{\mathrm{rm}}$-labeled probes, simultaneously monitoring those for RNaseP gene (an internal control) with its $\mathrm{VIC}^{\circ}$-labeled probe (ABI PRISM 7900HT Sequence Detection System and SDS v2.3 software, both Applied Biosystems). These CT values of the target gene and RNaseP gene were obtained for all the DNA samples. Copy number of the target gene was estimated from CT values by CopyCaller v1.0 software (Applied Biosystems).

\section{Statistics}

The ADM-2 algorithm prompted by Genomic Workbench software (edition 5.0.14, Agilent Technologies, 2010) was used to identify individual and common aberrations for 48 microarray data sets. This algorithm identifies all aberrant intervals with consistently high or low log ratios based on the statistical score. The algorithm searches for intervals where a statistical score based on the average qualityweighted log ratio of the sample and reference channels exceed a user-specified threshold. For the primary screening (Selection 1), we applied the following filtering options to the human genome assembly hg19 (excluding sex chromosomes): sensitivity threshold $=6$, fuzzy zero $=$ On, bin size $=10$, and centralization threshold $=6$. We then selected the primary candidate loci of somatic CNVs which exhibited $>=4$ difference in gain/loss calls in the whole penetrance summary.

To calculate mean signal OR and the probability of CNVs between groups, we plotted individual $\log 2$ signal ratios at all the probe sites within the above candidate loci. The Kolmogorov - Smirnov test revealed that $\log 2$ signal ratios were judged to fit into the Gaussian distribution at more than $80 \%$ of probe sites. Assuming their Gaussian distribution, we analyzed their statistical biases against $\log 2=0$ (i.e., the null hypothesis of equal signal intensities between patients and controls) by two tailed $t$-test at each probe position. Within a candidate $\mathrm{CNV}$ locus containing multiple probe sites, their $\log 2$ signal ratios were averaged and probabilities were summed and then subjected to Bonferroni's correction (Selection 2). Statistical difference of qPCR results between individual sample pairs was determined with ANOVA or two tailed $t$-test, incorporating technical errors into account. Alternatively, group differences of qPCR results from individual samples were estimated by the chi-square and MannWhitney U tests. Statistical analyses were performed using SPSS software (IBM Japan, Tokyo, Japan).

\section{Additional file}

Additional file 1: Figure S1. A flowchart of the study design. Table S1. Autopsy and clinical information of the subjects used. Table S2. List of candidate CNV regions and their statistical details. Table S3. Custom Taqman PCR primers and probes used.

\section{Abbreviations}

ADM: Aberration detection method; ANOVA: Analysis of variance; CGH: Comparative genomic hybridization; CNV: Copy number variation; CT: Cycle threshold; FISH: Fluorescent in situ hybridization; OR: Odds ratio; PMI: Postmortem interval; qPCR: Quantitative polymerase chain reaction.

\section{Competing interests}

The authors have no conflicts of interest to declare except OS; The author received honoraria from Otsuka Pharmaceutical; Shionogi; GlaxoSmithKline; Eli Lilly; and Tanabe Mitsubishi Pharma, and donations for research from the Otsuka Pharmaceutical Corporation.

\section{Authors' contributions}

TS, RK, and HN designed the experiments and wrote the manuscript; $M$. Sakai, YW, KA, M. Shibuya, AM, and HN performed the experiments; KN, KO, YK, HY, JM, AW, MH, TH, AH NK, SI, OS, KM, SN, HT, and AK collected and examined postmortem tissues. All authors read and approved the final manuscript.

\section{Acknowledgments}

This work was supported by Grant-in-Aids for Scientific Research on Innovative Areas (No. 24116010) and for Challenging Exploratory Research (No. 21659273) and a grant for Promotion of Niigata University Research Projects.

\section{Author details}

'Department of Molecular Neurobiology, Brain Research Institute, Niigata University, 1-757, Asahimachi-dori, 951-8585 Niigata, Japan. ${ }^{2}$ Department of Psychiatry, Graduate School of Medical and Dental Sciences, Niigata University, 1-757, Asahimachi-dori, 951-8510 Niigata, Japan. ${ }^{3}$ Matsuzawa Hospital, Setagaya-ku, 156-0057 Tokyo, Japan. ${ }^{4}$ Departments of Neuropsychiatry, Fukushima Medical University School of Medicine, 960-1295 Fukushima, Japan. ${ }^{5}$ Division of Psychiatry and Neurology, Kobe University Graduate School of Medicine, 650-0017 Kobe, Hyogo, Japan. 'Department of Mental Health, Nagoya University Graduate School of Medicine, 466-8550 Nagoya, Aichi, Japan. ${ }^{7}$ Department of Neuropsychiatry, Kinki University Faculty of Medicine, 589-8511 Osaka-Sayama, Osaka, Japan. ${ }^{8}$ Department of Social Rehabilitation, Kobe University School of Medicine, 654-0142 Hyogo, Japan. ${ }^{9}$ Department of Molecular Genetics, Brain Research Institute, Niigata University, 951-8585 Niigata, Japan. ${ }^{10}$ Pathology and Brain Disease Research Center, Brain Research Institute, Niigata University, 951-8585 Niigata, Japan.

Received: 9 March 2015 Accepted: 12 May 2015

Published online: 01 July 2015

\section{References}

1. Conrad DF, Pinto D, Redon R, Feuk L, Gokcumen O, Zhang Y, et al. Origins and functional impact of copy number variation in the human genome. Nature. 2010;464:704-12. 
2. Park H, Kim Jl, Ju YS, Gokcumen O, Mills RE, Kim S, et al. Discovery of common Asian copy number variants using integrated high-resolution array CGH and massively parallel DNA sequencing. Nat Genet. 2010;42:400-5.

3. Girirajan $\mathrm{S}$, Campbell CD, Eichle EE. Human copy number variation and complex genetic disease. Ann Rev Genetics. 2011;45:203-26.

4. Zhang F, Gu W, Hurles ME, Lupski JR. Copy number variation in human health, disease, and evolution. Annu Rev Genomics Hum Genet. 2009;10:451-81.

5. Malhotra D, Sebat J. CNVs: harbingers of a rare variant revolution in psychiatric genetics. Cell. 2012;148:1223-41.

6. Xu B, Roos JL, Levy S, van Rensburg EJ, Gogos JA, Karayiorgou M. Strong association of de novo copy number mutations with sporadic schizophrenia. Nat Genet. 2008;40:880-5.

7. Malhotra D, McCarthy S, Michaelson JJ, Vacic V, Burdick KE, Yoon S, et al. High frequencies of de novo CNVs in bipolar disorder and schizophrenia. Neuron. 2011;72:951-63.

8. Rees E, Moskvina V, Owen MJ, O'Donovan MC, Kirov G. De novo rates and selection of schizophrenia-associated copy number variants. Biol Psychiatry. 2011;70:1109-14

9. Kirov G, Pocklington AJ, Holmans P, Ivanov D, Ikeda M, Ruderfer D, et al. De novo CNV analysis implicates specific abnormalities of postsynaptic signalling complexes in the pathogenesis of schizophrenia. Mol Psychiatry. 2012:17:142-53.

10. Lourov IY, Vorsanova SG, Yurov YB. Molecular cytogenetics and cytogenomics of brain diseases. Curr Genomics. 2008:9:452-65.

11. Erickson RP. Somatic gene mutation and human disease other than cancer: an update. Mutat Res. 2010;705:96-106.

12. Lourov IY, Vorsanova SG, Yurov YB. Somatic cell genomics of brain disorders: a new opportunity to clarify genetic-environmental interactions. Cytogenet Genome Res. 2013;139:181-8.

13. Yurov YB, lourov IY, Vorsanova SG, Liehr T, Kolotii AD, Kutsev SI, et al. Aneuploidy and confined chromosomal mosaicism in the developing human brain. PLoS One. 2007;2:e558.

14. Yang AH, Kaushal D, Rehen SK, Kriedt K, Kingsbury MA, McConnell MJ, et al. Chromosome segregation defects contribute to aneuploidy in normal neural progenitor cells. J Neurosci. 2003;23:10454-62.

15. Piotrowski A, Bruder CE, Andersson R, Diaz de Ståhl T, Menzel U, et al. Somatic mosaicism for copy number variation in differentiated human tissues. Hum Mutat. 2008;29:1118-24.

16. De S. Somatic mosaicism in healthy human tissues. Trends Genet. 2011;27:217-23.

17. Lindhurst MJ, Parker VE, Payne F, Sapp JC, Rudge S, Harris J, et al. Mosaic overgrowth with fibroadipose hyperplasia is caused by somatic activating mutations in PIK3CA. Nat Genet. 2012;44:928-33.

18. Bloom RJ, Kähler AK, Collins AL, Chen G, Cannon TD, Hultman C, et al. Comprehensive analysis of copy number variation in monozygotic twins discordant for bipolar disorder or schizophrenia. Schizophr Res. 2013;146:289-90

19. Yurov YB, Vorsanova SG, lourov IY, Demidova IA, Beresheva AK, Kravetz VS, et al. Unexplained autism is frequently associated with low-level mosaic aneuploidy. J Med Genet. 2007;44:521-5.

20. Mosch B, Morawski M, Mittag A, Lenz D, Tarnok A, Arendt T. Aneuploidy and DNA replication in the normal human brain and Alzheimer's disease J Neurosci. 2007;27:6859-67.

21. Yurov YB, lourov IY, Vorsanova SG, Demidova IA, Kravets VS, Beresheva AK, et al. The schizophrenia brain exhibits low-level aneuploidy involving chromosome. Schizophr Res. 2008;98:139-47.

22. Iourov IY, Vorsanova SG, Liehr T, Yurov YB. Aneuploidy in the normal, Alzheimer's disease and ataxia-telangiectasia brain: differential expression and pathological meaning. Neurobiol Dis. 2009;34:212-20.

23. Iourov IY, Vorsanova SG, Liehr T, Kolotii AD, Yurov YB. Increased chromosome instability dramatically disrupts neural genome integrity and mediates cerebellar degeneration in the ataxia-telangiectasia brain. Hum Mol Genet. 2009;18:2656-69.

24. Lee JM, Zhang J, Su Al, Walker JR, Wiltshire T, Kang K, et al. A novel approach to investigate tissue-specific trinucleotide repeat instability. BMC Syst Biol. 2010:4:29.

25. Pamphlett R, Morahan JM, Luquin N, Yu B. Looking for differences in copy number between blood and brain in sporadic amyotrophic lateral sclerosis. Muscle Nerve. 2011;44:492-8.
26. Chen J, Cohen ML, Lerner AJ, Yang Y, Herrup K. DNA damage and cell cycle events implicate cerebellar dentate nucleus neurons as targets of Alzheimer's disease. Mol Neurodegener. 2010;5:60.

27. Arendt T, Brückner MK, Mosch B, Lösche A. Selective cell death of hyperploid neurons in Alzheimer's disease. Am J Pathol. 2010;177:15-20.

28. Ye T, Lipska BK, Tao R, Hyde TM, Wang L, Li C, et al. Analysis of copy number variations in brain DNA from patients with schizophrenia and other psychiatric disorders. Biol Psychiatry. 2012;72:651-4.

29. Shalon D, Smith SJ, Brown PO. A DNA microarray system for analyzing complex DNA samples using two-color fluorescent probe hybridization. Genome Res. 1996;6:639-45.

30. Yang YH, Speed T. Design issues for cDNA microarray experiments. Nat Rev Genet. 2002;3:579-88.

31. Zhang D, Qian Y, Akula N, Alliey-Rodriguez N, Tang J, Bipolar Genome Study, et al. Accuracy of CNV detection from GWAS data. PLoS One. 2011;6:e14511.

32. Ernst A, Alkass K, Bernard S, Salehpour M, Perl S, Tisdale J, et al. Neurogenesis in the striatum of the adult human brain. Cell. 2014;156:1072-83.

33. Williams SL, Mash DC, Züchner S, Moraes SC. Somatic mtDNA mutation spectra in the aging human putamen. PLoS Genet. 2013;9:10.

34. International Schizophrenia Consortium, Purcell SM, Wray NR, Stone JL, Visscher PM, O'Donovan MC, et al. Common polygenic variation contributes to risk of schizophrenia and bipolar disorder. Nature. 2009;460:748-52.

35. Filho AB, Souza J, Faucz FR, Sotomaior VS, Dupont B, Bartel F, et al. Somatic/ gonadal mosaicism in a syndromic form of ectrodactyly, including eye abnormalities, documented through array-based comparative genomic hybridization. Am J Med Genet A. 2011;155A:1152-6.

36. Etheridge SP, Bowles NE, Arrington CB, Pilcher T, Rope A, Wilde AA, et al. Somatic mosaicism contributes to phenotypic variation in Timothy syndrome. Am J Med Genet A. 2011;155A:2578-83.

37. Terracciano A, Specchio N, Darra F, Sferra A, Bernardina BD, Vigevano F, et al. Somatic mosaicism of $\mathrm{PCDH} 19$ mutation in a family with low-penetrance EFMR. Neurogenetics. 2012;13:341-5.

38. Bundo M, Toyoshima M, Okada Y, Akamatsu W, Ueda J, Nemoto-Miyauchi T, et al. Increased I1 retrotransposition in the neuronal genome in schizophrenia. Neuron. 2014;81:306-13.

39. Singer T, McConnell MJ, Marchetto MC, Coufal NG, Gage FH. LINE-1 retrotransposons: mediators of somatic variation in neuronal genomes? Trends Neurosci. 2010;33:345-54.

40. Peterson SE, Yang AH, Bushman DM, Westra JW, Yung YC, Barral S, et al. Aneuploid cells are differentially susceptible to caspase-mediated death during embryonic cerebral cortical development. J Neurosci. 2012;32:16213-22.

41. Yurov YB, Vostrikov VM, Vorsanova SG, Monakhov W, lourov IY. Multicolor fluorescent in situ hybridization on postmortem brain in schizophrenia as an approach for identification of low-level chromosomal aneuploidy in neuropsychiatric diseases. Brain Dev. 2001;23:S186-90.

42. Mkrtchyan H, Gross M, Hinreiner S, Polytiko A, Manvelyan M, Mrasek K, et al. The human genome puzzle - the role of copy number variation in somatic mosaicism. Curr Genomics. 2010;11:426-31.

43. lourov IY, Vorsanova SG, Yurov YB. Single cell genomics of the brain: focus on neuronal diversity and neuropsychiatric diseases. Curr Genomics. 2012;13:477-88.

44. Xu H, Poh WT, Sim X, Ong RT, Suo C, Tay WT, et al. SgD-CNV, a database for common and rare copy number variants in three Asian populations. Hum Mutat. 2011;32:1341-9.

45. Purcell SM, Moran JL, Fromer M, Ruderfer D, Solovieff N, Roussos P, et al. A polygenic burden of rare disruptive mutations in schizophrenia. Nature. 2014;506:185-90.

46. Priebe L, Degenhardt FA, Herms S, Haenisch B, Mattheisen M, Nieratschker V, et al. Genome-wide survey implicates the influence of copy number variants (CNVs) in the development of early-onset bipolar disorder. Mol Psychiatry. 2012;17:421-32.

47. Krumm N, O'Roak BJ, Karakoc E, Mohajeri K, Nelson B, Vives L, et al. Transmission disequilibrium of small CNVs in simplex autism. Am J Hum Genet. 2013:93:595-606.

48. Lencz T, Guha S, Liu C, Rosenfeld J, Mukherjee S, DeRosse P, et al. Genome-wide association study implicates NDST3 in schizophrenia and bipolar disorder. Nat Commun. 2013;4:2739.

49. Francey $L$, Conlin LK, Kadesch HE, Clark D, Berrodin D, Sun Y, et al. Genome-wide SNP genotyping identifies the Stereocilin (STRC) gene as a major contributor to pediatric bilateral sensorineural hearing impairment. Am J Med Genet A. 2012;158A:298-308. 
50. Pidsley R, Viana J, Hannon E, Spiers HH, Troakes C, Al-Saraj S, et al. Methylomic profiling of human brain tissue supports a neurodevelopmental origin for schizophrenia. Genome Biol. 2014;15:483.

51. Huang KC, Yang KC, Lin H, Tsao Tsun-Hui T, Lee WK, Lee SA, et al. Analysis of schizophrenia and hepatocellular carcinoma genetic network with corresponding modularity and pathways: novel insights to the immune system. BMC Genomics. 2013;14 Suppl 5:S10.

52. Shanmuganathan A, Black MB. Researchgate. 2014. http://www.researchgate.net/ post/What_is_the_fold_change_value_correlation_between_microarray_and_ qPCR_experiments

53. Tsubahara M, Hayashi Y, Niijima S, Yamamoto M, Kamijo T, Murata Y, et al. Isolated growth hormone deficiency in two siblings because of paternal mosaicism for a mutation in the GH1 gene. Clin Endocrinol (Oxf). 2012;76:420-4

54. Kurek KC, Luks VL, Ayturk UM, Alomari Al, Fishman SJ, Spencer SA, et al. Somatic mosaic activating mutations in PIK3CA cause CLOVES syndrome. Am J Hum Genet. 2012:90:1108-15.

55. Abyzov A, Mariani J, Palejev D, Zhang Y, Haney MS, Tomasini L, et al. Somatic copy number mosaicism in human skin revealed by induced pluripotent stem cells. Nature. 2012;492:438-42.

56. Whale AS, Huggett JF, Cowen S, Zhang Y, Haney MS, Tomasini L, et al. Comparison of microfluidic digital PCR and conventional quantitative PCR for measuring copy number variation. Nucleic Acids Res. 2012;40:e82.

57. Watanabe Y, Nunokawa A, Kaneko N, Someya T. A case-control study and meta-analysis of association between a common copy number variation of the glutathione S-transferase mu 1 (GSTM1) gene and schizophrenia. Schizophr Res. 2010;124:236-7.

\section{Submit your next manuscript to BioMed Central and take full advantage of:}

- Convenient online submission

- Thorough peer review

- No space constraints or color figure charges

- Immediate publication on acceptance

- Inclusion in PubMed, CAS, Scopus and Google Scholar

- Research which is freely available for redistribution 